\title{
Microstructure and Time-Dependent Behavior of STx-1b Calcium Montmorillonite Suspensions
}

\author{
Yee-Kwong Leong • Pengfei Liu • Pek-Ing Au • \\ Peta Clode $\cdot$ Jishan Liu
}

Accepted: 15 December 2021

(C) The Author(s) 2022

\begin{abstract}
CaMnt is much less important than NaMnt due to its limited commercial applications. The time-dependent property of NaMnt gel has been studied extensively as it is exploited in many applications such as drilling mud and viscosity-modifier applications. In contrast, the time-dependent property of CaMnt suspension and the factors affecting it are largely unknown. The speciation of $\mathrm{Ca}^{2+}$ ions is one such factor to be evaluated. In the current study, $\mathrm{pH}$ and solids concentration were examined and then used to validate a recent theory on clay gel time dependency. The results supported the theory that a strong electrostatic double layer (EDL) repulsion in the 3-D network is needed to reorganize the structure and drive it toward the state of minimum free energy. The 12 wt.\% CaMnt (STx-1b) gel displayed timedependent behavior at $\mathrm{pH} 5$ but not at its natural $\mathrm{pH}$ of 8.4. At $\mathrm{pH} 5$, the interlayer $\mathrm{Ca}^{2+}$ ions became fully hydrated and desorbed from the platelet surface. This enhanced the EDL repulsion between the platelets. At
\end{abstract}

Y.-K. Leong $(\bowtie) \cdot$ P. Liu $\cdot$ P.-I. Au $\cdot$ J. Liu

Department of Chemical Engineering, The University of Western Australia, 35 Stirling Highway, Crawley,

WA 6009, Australia

e-mail: yeekwong.leong@uwa.edu.au

P. Clode

Centre for Microscopy, Characterization and Analysis, The University of Western Australia, 35 Stirling Highway, Crawley, WA 6009, Australia
$\mathrm{pH}>6.5$, the positively charged hydrolysis product $\mathrm{Ca}(\mathrm{OH})^{+}$began to form and was adsorbed, partially neutralizing the permanent layer charge and weakening the repulsion. However, at 19.4 wt.\% solids, the gel displayed pronounced time-dependent behavior despite the high natural $\mathrm{pH}$ of 8 . The platelets were much closer together, allowing the EDL force to operate and effect structural reorganization. The microstructure of these CaMnt gels showed high platelet concentrations interacting to form a relatively open structure. The microstructure of a kaolin (KGa-2) suspension which showed no time-dependent behavior even after 1 day of ageing revealed the importance of particle morphology and layer charge on time dependency. Its 3-D structure was formed by relatively thick, layered platelets with a low layer charge.

Keywords Hydrolysis Products · Kaolin · Montmorillonite · Time-dependent mechanisms · Yield stress $\cdot$ Zeta potential

\section{Introduction}

The time-dependent behavior of calcium montmorillonite (CaMnt) gels characterized by an increasing yield stress with ageing time has not been researched thoroughly and so knowledge gaps exist. In contrast, the ageing or gelation behavior of bentonite and sodium montmorillonite (NaMnt) have been examined extensively (Broughton \& Squires, 1936; Callaghan \& Ottewill, 1974; Hauser \& Reed, 1937; M'Ewen \& Pratt, 1957; van Olphen, 1955), with this behavior being first reported almost 100 years ago. 
These gels displayed pronounced thixotropic behavior at a very low concentration of a few percent solids. Surface chemistry factors, such as $\mathrm{pH}$ and salt concentration, are important gelation factors for bentonite or NaMnt (Abend \& Lagaly, 2000; Brandenburg \& Lagaly, 1988; Heller \& Keren, 2001; Tombacz \& Szekeres, 2004). CaMnt gels were reported to display time-independent behavior (Keren, 1988; Sueto \& Nakaishi, 1992) and pH-independent rheological behavior at a low concentration of a few percent solids (Keren, 1988). Sueto and Nakaishi (1992) also found that their CaMnt gel did not display timedependent behavior over a wide salt concentration of between $0.001 \mathrm{M}$ and $1.0 \mathrm{M} \mathrm{NaCl}$. They used the flow hysteresis loop method to determine the time-dependent behavior. Abu-Jdayil (2011), however, reported a relatively weak time-dependent behavior for unpurified CaMnt gel at $6 \mathrm{wt} \%$ solids as reflected by an increasing yield stress with ageing time. The yield stress was derived from constitutive models used to fit the flow data. This CaMnt, used in drilling mud formulation, has a relatively high $\mathrm{Na}_{2} \mathrm{O} / \mathrm{CaO}$ ratio of 0.8 , suggesting the presence of NaMnt. The $\mathrm{Na}_{2} \mathrm{O}$ and $\mathrm{CaO}$ contents were 3.215 wt.\% and 3.986 wt.\%, respectively. The experimental protocol employed is not ideal for a study of ageing. The structural state of the aged gel will have suffered damage during: (1) its transfer to the rheometer; and (2) the preshearing step adopted prior to each flow characterization. This could be the reason for the very short ageing time of $24 \mathrm{~h}$ reported to attain the fully recovered state. This is in contrast to an ageing time of months and years which was reported for three different thixotropic clay gels (Leong et al., 2021). Leong et al. (2021) also discussed in detail the various methods for characterization of time-dependent behavior and why the results are usually not quantitatively comparable. One of the reasons is that the structural state of the gel at the start of the time-dependent experiment was not well defined. The sparse investigation of CaMnt gel rheology is due to its limited commercial applications (Choo et al., 2020). One of the knowledge gaps in factors affecting the time-dependent rheology is the state or speciation of $\mathrm{Ca}^{2+}$ ions. At low $\mathrm{pH}, \mathrm{Ca}^{2+}$ is fully hydrated as $\mathrm{Ca}\left(\mathrm{H}_{2} \mathrm{O}\right)_{6}{ }^{2+}$ and this ionic species appeared not to adsorb. At alkaline $\mathrm{pH}$ it formed the hydrolysis product $\mathrm{Ca}(\mathrm{OH})^{+}$which was adsorbed readily onto the particle surface, affecting the zeta potential and rheology of the suspension (Zhu et al.,
2016). The strength of the platelet-platelet interactions should, therefore, be affected by the nature of the $\mathrm{Ca}^{2+}$ ions. This postulate was examined in the current study in which the time-dependent behavior of CaMnt (STx-1b) gels was evaluated as a function of $\mathrm{pH}$ and solids concentration. The $\mathrm{pH}$ chosen was such that the $\mathrm{Ca}^{2+}$ ions were in either the fully hydrated or the hydrolysis product form. The microstructures of these gels were also captured and their relationship with the time-dependent behavior discussed.

Leong et al. (2018) and Du et al (2018) identified the conditions required for time-dependent behavior. These conditions are a 3-D network structure formed by heterogeneous charge attraction between negative faces and positive edges (plus contributions from van der Waals attractive force) and a strong face-face repulsion between platelets in the structure. The strong face-face electrostatic double layer (EDL) repulsion governs the development of the microstructure and is responsible for the time-dependent behavior. The EDL repulsive force causes the platelets to break apart, orientate, move, and reform stronger bonds to produce a stronger network structure with time. The structure moves progressively towards a lower free energy state, the driving force for the time-dependent behavior. The platelet charge properties, therefore, have an important effect. The $\mathrm{pH}$ was shown to have a pronounced effect on the thixotropic and ageing behavior of NaMnt and bentonite gel (Lee et al., 2012; Tombacz \& Szekeres, 2004). Low pH tended to produce a stronger effect, as it gives rise to a stronger edge-face attraction as a result of a higher positive charge density of the edge. Face-face repulsion remained strong as the negative layer charge was permanent. A stronger network structure thus forms with time. The effect of layer charge density on the gel time-dependent property is, however, unclear. The use of CaMnt will help provide a better understanding of this effect by exploiting the different adsorption capacity of $\mathrm{Ca}\left(\mathrm{H}_{2} \mathrm{O}\right)_{6}{ }^{2+}$ and its hydrolysis product $\mathrm{Ca}(\mathrm{OH})^{+}$. The effects of these $\mathrm{Ca}^{2+}$ species on the time-dependent rheology of CaMnt gel should reveal definitive information on the effect of the layer charge.

Ice crystals were believed to have affected the microstructure of STx-1b CaMnt gels obtained by cryo-SEM imaging published by $\mathrm{Au}$ and Leong (2016). These hexagonal crystals fractured the clay gel microstructure, producing large pores and 
channels (Au et al., 2015a; Au \& Leong, 2016; Du et al., 2018; Leong et al., 2018). The purpose of the present study, therefore, was to obtain artefact-free microstructures of STx-1b CaMnt gels by preventing ice-crystal formation by means of subjecting the gel samples to very high pressure and ultra-fast cryofreezing (Du et al., 2018; Leong et al., 2018; Wyss et al., 2004).

The microstructure and time-dependent properties of high-defect $\mathrm{KGa}-2$ kaolin suspensions will be examined also for comparison purposes. The morphology and charge properties of kaolin particles are very different from CaMnt platelets. Yet, under certain conditions, both clay suspensions will be shown to display time-independent ageing behavior.

\section{Materials and Methods}

The STx-1b CaMnt was obtained from the Source Clays Repository of The Clay Minerals Society (CMS). The clay was sourced from the Manning Formation located in the county of Gonzales, Texas. The physical and chemical data of STx-1 are given in the CMS database for source clays. The physical and chemical properties of STx-1 and STx-1b are quite similar. The major exchangeable cation is $\mathrm{Ca}^{2+}$. STX-1 has a BET surface area of $83.8 \mathrm{~m}^{2} / \mathrm{g}$ and a CEC of $84.4 \mathrm{meq} / 100 \mathrm{~g}$. It has a high octahedral charge of -0.68 e per formula unit. The tetrahedral sheet does not have a permanent charge. $\mathrm{CaO}$ and $\mathrm{MgO}$ contents are 1.59 and $3.69 \%$, respectively. The isomorphic substitution of the $\mathrm{Al}^{3+}$ ion in the octahedral sheet by a divalent metal ion such as $\mathrm{Mg}^{2+}$ is responsible for the high permanent charge. It has a low $\mathrm{Na}_{2} \mathrm{O} / \mathrm{CaO}$ ratio of 0.17. Impurities present are quartz, silica, and carbonate minerals. KGa-2 kaolin, also sourced from CMS, has a zero tetrahedral charge and a relatively low positive octahedral charge of 0.16 e. Isomorphic substitution of $\mathrm{Al}^{3+}$ by $\mathrm{Ti}^{4+}$ in the octahedral sheet is the likely cause. This kaolin has a BET surface area of $23.5 \mathrm{~m}^{2} / \mathrm{g}$ and a CEC of $3.3 \mathrm{meq} / 100 \mathrm{~g}$.

The CaMnt was purified by sonication, sedimentation, and decantation, collecting the supernatant. A very dilute suspension of $1 \mathrm{wt} . \%$ solids was used and the CaMnt was dispersed with a sonic probe. The impurities were left to settle to the bottom of the container and the supernatant containing the $\mathrm{CaMnt}$ platelets was collected and dried in an oven at $105^{\circ} \mathrm{C}$.
The purified CaMnt was used in the gel preparation for this study. The suspensions prepared were relatively concentrated, 12-25 wt.\% solids. Each suspension, weighing $\sim 40 \mathrm{~g}$, was prepared in DI water by sonication with a sonic probe for $\sim 2 \mathrm{~min}$. The prepared suspensions were left to rest for at least a week before the experimentation. This was to ensure the particles or platelets have time to attain physicochemical equilibrium. The yield stress in the ageing study was measured directly with either a Brookfield RVDV-II + PRO or a LVDV-II + PRO vane viscometer (AMETEK Brookfield, Middleboro, Massachusetts, USA). In this measurement, a four-blade vane (diameter $0.5 \mathrm{~cm}$, height $1.0 \mathrm{~cm}$ ) was immersed in the suspension and rotated slowly at $0.6 \mathrm{rpm}$. The maximum torque used in the yield stress calculation was recorded. In the ageing experiment, the gel was first mixed thoroughly for 2 min with a spatula and then allowed to rest undisturbed until the predetermined time for the yield stress measurement. The vane measurement was conducted in an area not disturbed by a previous measurement. The zeta potential was measured with a ZetaProbe (Colloidal Dynamics Inc., Ponte Vedra Beach, Florida, USA) operated in a potentiometric titration mode, as a function of decreasing or increasing $\mathrm{pH}$. The step-down shear rate rheology was performed in a $1^{\circ}$ cone-and-plate MCR72 control rate rheometer (Anton Paar GmbH, Graz, Austria) at an ambient temperature of $22^{\circ} \mathrm{C}$. The gel was first sheared at a high constant shear rate of $1000 \mathrm{~s}^{-1}$ until equilibrium was reached as reflected by a constant shear stress response, and then abruptly stepped down to $10 \mathrm{~s}^{-1}$. The shear stress was monitored.

The gel microstructures were imaged at $5 \mathrm{kV}$ with a Zeiss 55 field emission SEM fitted with a Leica EM VCT100 cryo and anti-contamination system (Carl Zeiss Microscopy GmbH, Jena, Germany). The gel samples for imaging were first subjected to high pressure of 2000 bar and then frozen rapidly at a rate of $25,000^{\circ} \mathrm{C} \mathrm{s}^{-1}$. The samples were then sublimated and coated with a $7 \mathrm{~nm}$ thick film of Pt before imaging.

\section{Results and Discussion}

The ageing of 12.3 wt.\% purified CaMnt gels at their natural $\mathrm{pH}$ of 8.4 and at $\mathrm{pH} \sim 5$ (4.5-5.3) displayed very dissimilar behaviors (Fig. 1). The gel at 


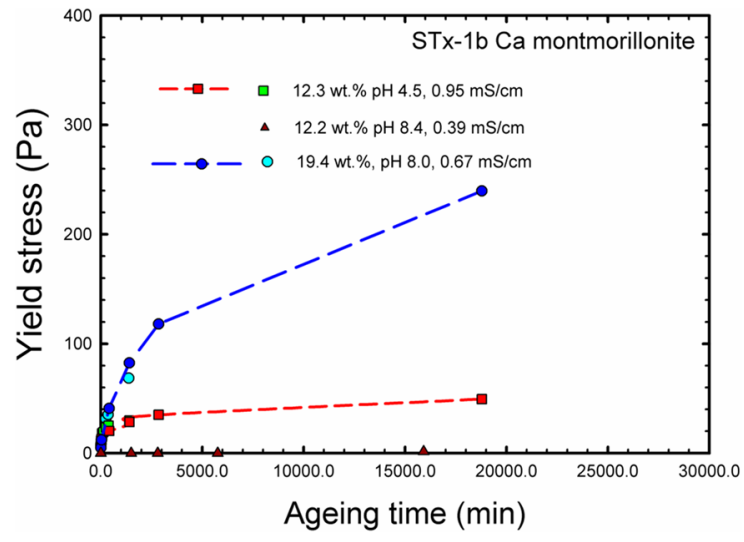

Fig. 1 The ageing behavior of CaMnt gels at various $\mathrm{pH}$ and solids loading

its natural $\mathrm{pH}$ did not exhibit time-dependent behavior; the yield stress remained essentially unchanged even after 4 days of ageing. The yield stress was close to $0 \mathrm{~Pa}$. The lower $\mathrm{pH}$ gel, however, demonstrated pronounced ageing behavior. The yield-stress increase was rapid at the start of ageing. The rate of increase slowed considerably after the first day. The yield stress of $9 \mathrm{~Pa}$ after $1 \mathrm{~min}$ of ageing increased to $50 \mathrm{~Pa}$ after $18,787 \mathrm{~min}$ (13 days) of rest, a 5-fold increase. At $\mathrm{pH} 8.4$, the $\mathrm{Ca}^{2+}$ ions existed as a positively charged hydrolysis product $\mathrm{Ca}(\mathrm{OH})^{+}($Baes \& Mesmer, 1986), according to the zeta potential-pH results obtained for alumina suspension at various $\mathrm{CaCl}_{2}$ concentrations (Zhu et al., 2016). This hydrolysis product $\mathrm{Ca}(\mathrm{OH})^{+}$adsorbed readily on the clay platelets, thus reducing the value of its negative face charge density, weakening the face-face repulsion. This condition was, thus, not conducive for the timedependent behavior to occur. At $\mathrm{pH}<6$, all the $\mathrm{Ca}^{2+}$ ions were in the hydrated form with 6 to 12 water molecules per cation (Rudolph \& Irmer, 2013; TeichMcGoldrick et al., 2015; Zavitsas, 2005). These bulky hydrated ions were unable to adsorb strongly and would spend most of their time in the diffuse layer. The negative face-charge density increased, therefore, producing stronger face-face repulsion that could break bonds and move and orientate platelets to form a stronger structure.

The zeta potential provides information on the dominant charge determining the surface properties of a clay. It cannot be used as a measure of the strength of the particle-particle repulsive interaction

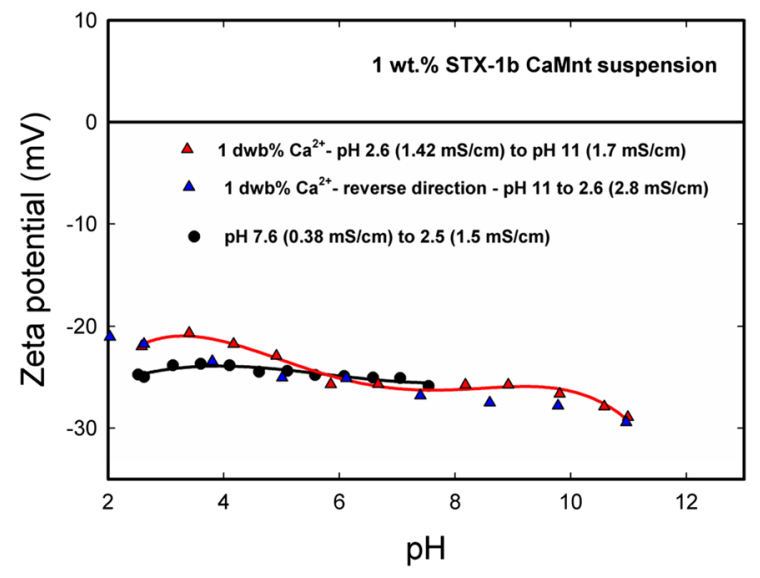

Fig. 2 The zeta potential-pH behavior of STx-1b CaMnt suspensions with and without added $\mathrm{CaCl}_{2}$. The dosage of $\mathrm{Ca}^{2+}$ added was $1 \mathrm{dwb} \%$ ( $\mathrm{g} \mathrm{Ca}^{2+} / 100 \mathrm{~g} \mathrm{CaMnt}$ solids)

because of the localized heterogeneous charge properties of the clay platelets. Both the layer or face and edge charges and their interactions with the diffuse layer ions contribute to the zeta potential. Adsorbed charged additives also affect this zeta potential.

The zeta potential data of $1 \mathrm{wt} . \%$ CaMnt suspension prepared from the 19.4 wt.\% gel (Fig. 2) were all negative from $\mathrm{pH} 2.5$ to 8 and showed a weak dependence on $\mathrm{pH}$. The permanent negative charge in the octahedral sheet dominated the CaMnt net surface charge at all $\mathrm{pH}$ values. The magnitude of $-25.9 \mathrm{mV}$ at $\mathrm{pH} 7.6$ and electrical conductivity of $0.4 \mathrm{mS} / \mathrm{cm}$ decreased to $-25 \mathrm{mV}$ at $\mathrm{pH} 2.6$ and $1.24 \mathrm{mS} / \mathrm{cm}$. The high proton mobility and concentration at $\mathrm{pH} 2.6$ contributed to the high conductivity. The initial solution ionic strength was relatively low as reflected by a conductivity of $0.4-0.45 \mathrm{mS} / \mathrm{cm}$, which is equivalent to a $\mathrm{KCl}$ concentration of only $\sim 0.003 \mathrm{M}$. Addition of $0.1 \mathrm{~g} \mathrm{Ca}^{2+} / 100 \mathrm{~g} \mathrm{CaMnt}$ or $0.1 \mathrm{dwb} \% \mathrm{Ca}^{2+}$ (dwb -dry weight basis) at $\mathrm{pH} 2.5$ caused the $\mathrm{pH}$ to increase slightly to 2.6 and the negative zeta potential value to decrease to $-22 \mathrm{mV}$, representing a $12 \%$ reduction. The corresponding conductivity was increased by $14 \%$ but this change was regarded as small for a 'normal' ionic strength-effect study where orders of magnitude change in salt concentration are normally employed. The smaller zeta potential was due to the effect of solution-based $\mathrm{Ca}\left(\mathrm{H}_{2} \mathrm{O}\right)_{6}{ }^{2+}$ shielding the surface charge. Between $\mathrm{pH} 6$ and 9 the zeta potential was essentially constant and so was the conductivity, $1.12-1.2 \mathrm{mS} / \mathrm{cm}$. The positively charged hydrolysis product $\mathrm{Ca}(\mathrm{OH})^{+}$ 
began to form at $\mathrm{pH} \sim 6.5$ and increased with $\mathrm{pH}$. The constant zeta potential suggested its increased formation and adsorption being compensated exactly by a corresponding increase in the concentration of the edge $\mathrm{pH}$-dependent charge sites acquiring more negative charges or becoming neutral. The relatively small effect of $0.1 \mathrm{dwb} \% \mathrm{Ca}^{2+}$ on the zeta potential was due to an already high $\mathrm{Ca}^{2+}$ content existing as interlayer ions in the STx-1b. The $\mathrm{Ca}^{2+}$ content of STx-1 was 1.14 wt.\%, or in terms of $\mathrm{CaO}, 1.59 \mathrm{wt} . \%$, of the clay according to the source clay data. A 7 wt.\% bentonite suspension in $1 \mathrm{M} \mathrm{CaCl}_{2}(112 \mathrm{mS} / \mathrm{cm})$ was reported to display a negative zeta potential of $-18 \mathrm{mV}$ at $\mathrm{pH} 7.2$ (Au et al., 2015b), which is another example showing the dominant influence of the negative layer charge on the platelet surface property. For suspensions with negligible $\mathrm{Ca}^{2+}$ present, the effect of $\mathrm{Ca}^{2+}$ on zeta potential at $\mathrm{pH}$ above 6.5 was quite pronounced such as in the case of alumina suspensions (Zhu et al., 2016). $\mathrm{Ca}^{2+}$ caused the trend of the zeta potential-pH behavior to deviate sharply at this $\mathrm{pH}$.

The microstructure of the thixotropic 12.3 wt.\% CaMnt gel at $\mathrm{pH} \sim 5$ (Fig. 3) showed irregularly shaped, flexible, $\sim 1 \mu \mathrm{m}$ platelets with a curling feature, forming an open 3-D network structure. The platelets were well separated with most adopting an almost vertical orientation. The EDL repulsive force between these platelets in the face-face configuration was responsible for opening up the structure. Heterogeneous charge and van der Waals attraction between the face and edge formed the platelet-platelet bonds and network junctions. The structure showed pores

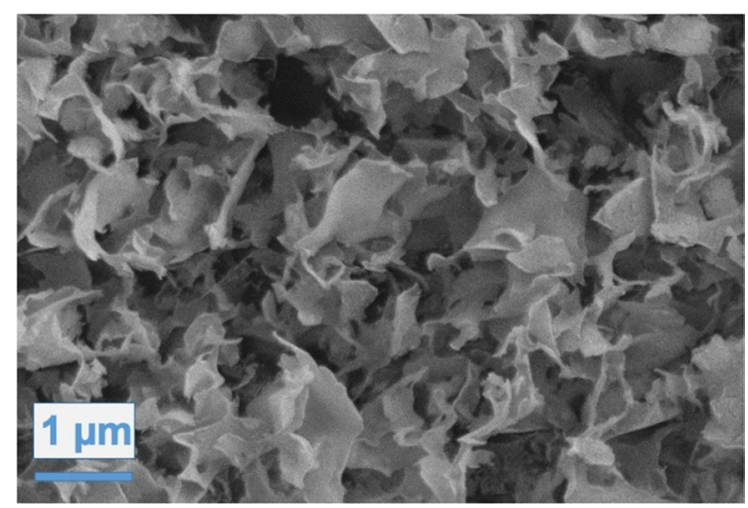

Fig. 3 The microstructure of thixotropic 12.3 wt.\% STx-1b CaMnt at pH 5.32 and conductivity of $0.97 \mathrm{mS} \mathrm{cm}^{-1}$ aged for 3 months. This conductivity is equivalent to $0.007 \mathrm{M} \mathrm{KCl}$ being quite large in diameter and deep. The EDL repulsive force must be controlling the development of the 3-D structure and driving it towards a minimum free energy state (Du et al., 2018; Leong et al., 2018). This state was achieved by the platelets undergoing processes of bond breaking, orientating, moving, and establishing stronger bonds. The strength of the structure reflects the free energy state of the gel. The stronger the structure, the lower its free energy.

At the higher CaMnt concentration of 19.4 wt.\%, the suspension also displayed time-dependent behavior (Fig. 1) despite its high $\mathrm{pH}$ of 8.0. The
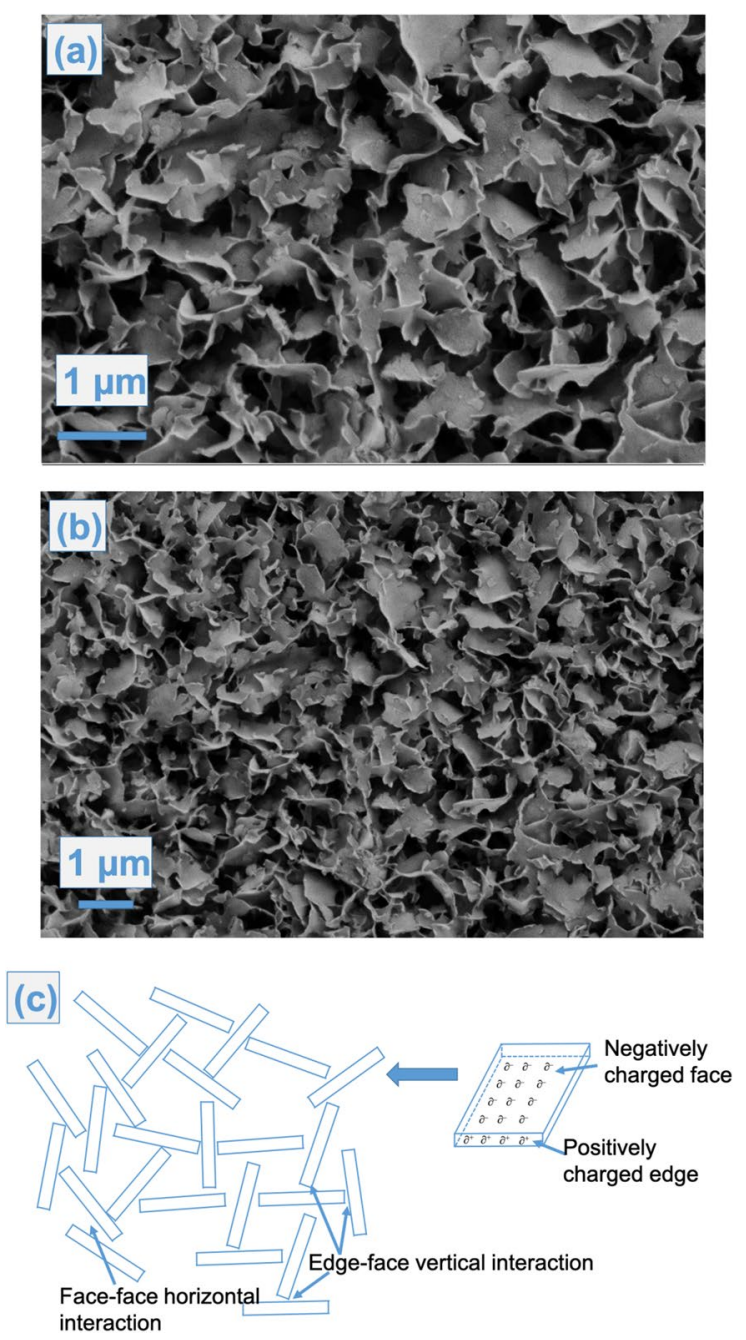

Fig. 4 The microstructure of thixotropic 19.4\% CaMnt gel at pH 8 with conductivity $0.77 \mathrm{mS} / \mathrm{cm}$ aged for 3 months imaged at different magnifications 
yield stress increased from $5.4 \mathrm{~Pa}$ at 1 min ageing to $240 \mathrm{~Pa}$ after 13 days. This was an enormous, 44-fold increase. Its corresponding microstructure (Fig. 4a) captured at the same magnification of 12.3 wt.\% gel at $\mathrm{pH} \sim 5$ showed an open structure with a much higher platelet density and smaller pores. Many of the platelets were interacting both attractively and repulsively in a near-vertical orientation forming the open structure (Fig. 4a, b). A schematic of the platelet-platelet interactions forming the open structure, viewed from the top is shown in Fig. 4c. The pore diameters were relatively small but appeared to be deep. The EDL repulsive force governed the development of the 3-D structure and drove it towards a minimum free energy state despite a lower negative charge density of the face, a result of partial neutralization by the positively charged $\mathrm{Ca}^{2+}$ hydrolysis products. The smaller face-face separation distance brought the platelets to within the operational range of the weakened EDL repulsive force.

The microstructure of an even more concentrated CaMnt suspension at $25 \mathrm{wt} \%$ solids in Fig. 5a and at a higher magnification in Fig. 5b showed a relatively large concentration of interacting platelets adopting a flat-lying configuration. The solids volume fraction was only 0.12 . Some of the pores or void spaces are highlighted by red crosses (Fig. 5b). Each platelet in the structure has more neighbors within the operational range of their repulsive and attractive forces. The configuration adopted is, thus, the result of the complex interactions of these forces with all their nearest neighbors. At high platelet concentration, adopting a vertical orientation was, thus, not possible for many platelets. This suspension displayed a yield stress of $56.3 \mathrm{~Pa}$. Its $\mathrm{pH}$ and conductivity of 8 and $0.87 \mathrm{mS} / \mathrm{cm}$ were similar to that obtained for the 19.4 wt.\% suspension. A few compact agglomerates can also be seen clearly in Fig. 5b. In contrast to the NaMnt platelets in Fig. 5c, the CaMnt platelets appeared to be thicker and more rigid. These CaMnt platelets were likely to be a few layers thick.

Despite the low solids volume fraction of 0.12 , the high density of irregularly-shaped platelets, mostly $\sim 1$ $\mu \mathrm{m}$ in size (Fig. 5 and b), was due to platelets interacting at different depth levels being projected onto the 2-D image. The many flat-lying platelets interacted with others in all configurations, lying in flat and upright vertical positions at all angles, to form
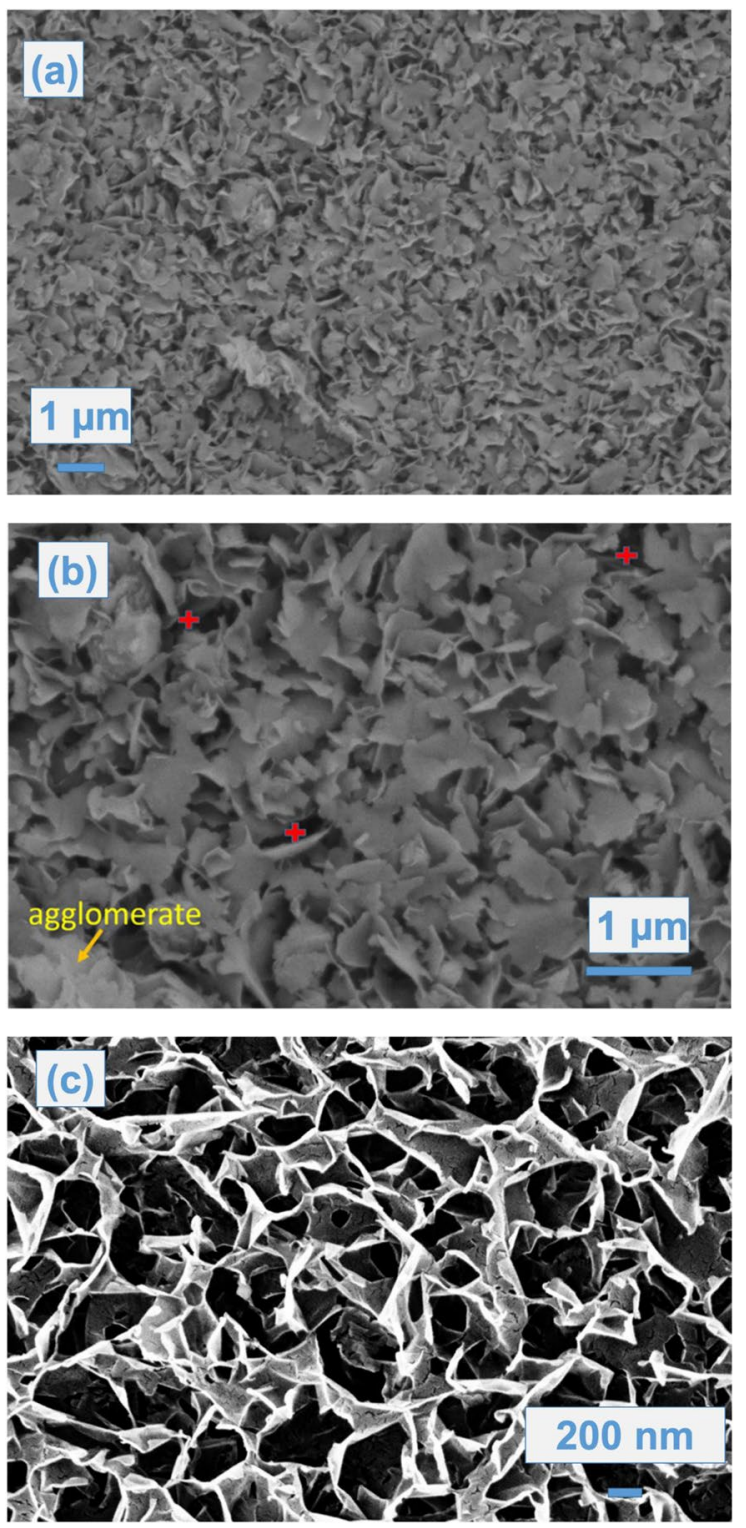

Fig. 5 The microstructure of 25 wt.\% STx-1b CaMnt suspension imaged at a magnification of a $20 \mathrm{kX}$ and $\mathbf{b} 50 \mathrm{kX}$, and $\mathbf{c}$ the microstructure of 4 wt. $\%$ NaMnt (SWy-2) at $80 \mathrm{kX}$

the 3-D structure. Curly and irregularly shaped edges enhanced the opportunity to form bonds and network junctions. Most pores were obscured by platelets lying flat but some of the observable deep ones are highlighted by red crosses in Fig. 5 b.

In contrast, the microstructure of 4 wt.\% NaMnt gel (Fig. 5c) showed thinner and more flexible platelets. These platelets were a single layer thick (Lagaly, 
2006). The strong face-face EDL repulsion, edgeface attraction, and high platelet concentration forming the network structure were responsible for the high yield stress and the pronounced time-dependent behavior displayed by the gel at a few weight percent solids (Leong et al., 2018). It is thus not possible to produce a homogeneous, handleable NaMnt suspension at $25 \mathrm{wt} . \%$ solids as the yield stress will be infinite. In contrast, the layered CaMnt platelets with their reduced negative charge density caused by adsorbed $\mathrm{Ca}^{2+}$ hydrolysis products required a much higher concentration to display appreciable yield stress and time-dependent behavior.

Kaolin particles in suspension also displayed weak face-face repulsion. The $38.5 \mathrm{wt}$ \% KGa-2 kaolin suspension at $\mathrm{pH} 5.2$ with a conductivity of $2.03 \mathrm{mS} / \mathrm{cm}$ (Fig. 6) did not display any time-dependent behavior after 1 day of ageing. The yield stress remained unchanged, 212 and $210 \mathrm{~Pa}$ at $3 \mathrm{~min}$ and $23 \mathrm{~h}$ of ageing. The microstructure and particle morphology of the KGa-2 suspension were very different from that found in the CaMnt gel. These differences in timedependent, particle and microstructure properties were used to validate the theory on the mechanism or cause of immediate or undelayed thixotropy (Leong et al., 2018, 2021).

The microstructure of a 26.5 wt. $\%$ or 12.2 vol. $\%$ kaolin suspension at $\mathrm{pH} 4.9$ with a yield stress of 78 Pa (Fig. 7a and b) was formed by bulky, multilayered, irregularly shaped clay particles. The edges were jagged with platelets of various sizes bonded together

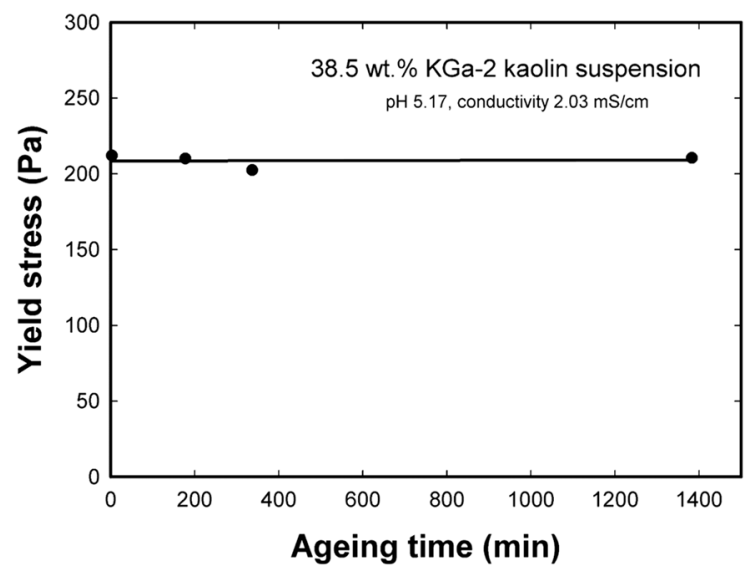

Fig. 6 Ageing behavior of high-defect $\mathrm{KGa}-2$ kaolin suspension showing the absence of time-dependent behavior
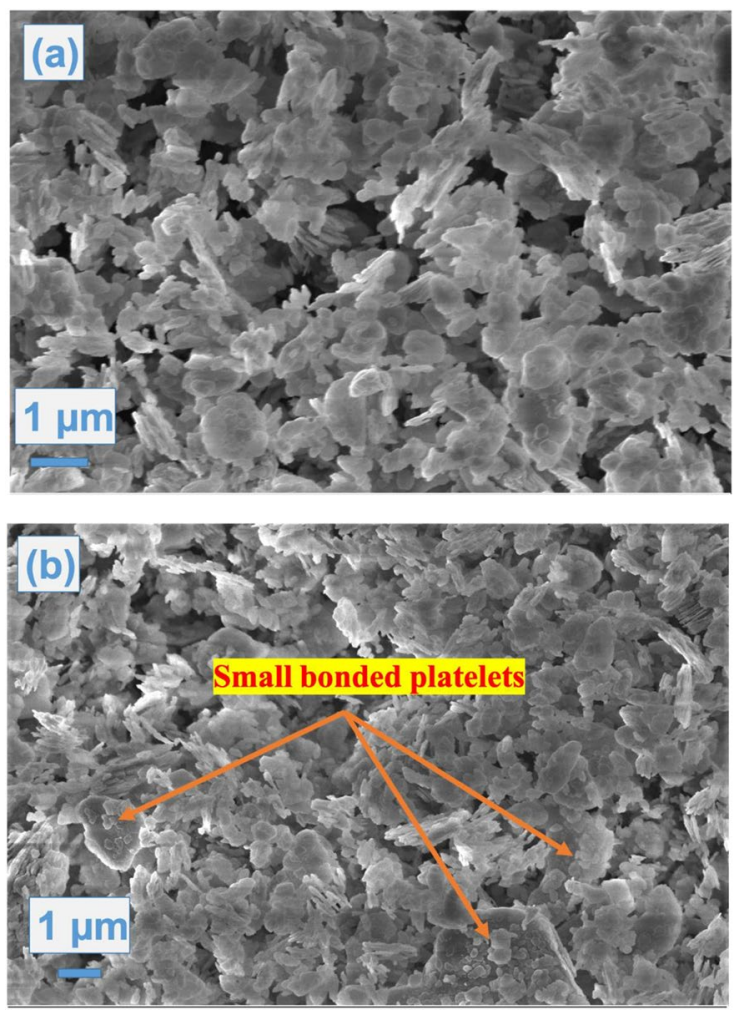

Fig. 7 The microstructure of high-defect 26.5 wt.\% KGa-2 kaolinite suspension imaged at a $30 \mathrm{kX}$ and b $20 \mathrm{kX}$

in layers to form the particles. This increased the edge area relative to the face area compared to that of a single unattached platelet. The edge charge sites will also be high. For many of these particles, a few small bonded platelets formed the outermost face (red arrows in Fig. 7b). These particles with a small outer face area are not conducive to producing strong face-face repulsion. The kaolin has a small (positive) layer charge, 0.16 e per formula unit, located in the alumina octahedral sheet. The EDL repulsive interaction for all kaolin particles in the network is unlikely to be strong. An essential condition for time-dependent behavior is, therefore, not met.

Many studies have examined the time-dependent properties of clay gels between different sheardependent equilibrium states. One study employed a stepdown constant-stress approach in which the gel was sheared at a high constant stress until a constant shear rate was attained and then stepped down abruptly to a lower stress and the shear rate was monitored continuously (Coussot et al., 2002, 2006). 
After stepdown, the shear rate decreased as the structure recovered to the equilibrium state of the applied constant stress. Sometimes the flow stopped, i.e. the shear rate decreased to zero. This occurred when the recovered structure had a yield stress greater than the applied shear stress. A similar approach can be used to determine whether a suspension is time dependent. This was the stepdown constant shear rate approach with the shear stress being monitored as a function of time (Leong et al., 2021). The gel was subjected to a high constant shear rate until the equilibrium state was reached which was reflected by a constant shear stress response. After that the shear rate stepped down abruptly to a lower value. The high shear equilibrium structure will reorganize upon stepdown to produce a stronger equilibrium structure associated with the low shear rate. This process takes time and was reflected by an increasing shear stress commencing immediately upon stepdown. In the present study, the gel was sheared at a constant rate of $1000 \mathrm{~s}^{-1}$ to an equilibrium state and then stepped down to a lower rate of $10 \mathrm{~s}^{-1}$. A $1^{\circ}$ cone-plate viscometer was used so that everywhere in the gel in the gap experienced the same shear rate.

The stepdown shear-rate results for 19.4 wt.\% CaMnt and 38.5 wt.\% kaolin suspensions (Fig. 8) showed very different behaviors. The shear stress of the $19.4 \mathrm{wt} \%$ CaMnt gel displayed immediate increase upon stepdown to $10 \mathrm{~s}^{-1}$. It was still increasing after $3 \mathrm{~min}$ of shearing. This is a reflection of the slow kinetics of the structural reorganization process. At $1000 \mathrm{~s}^{-1}$, the equilibrium state

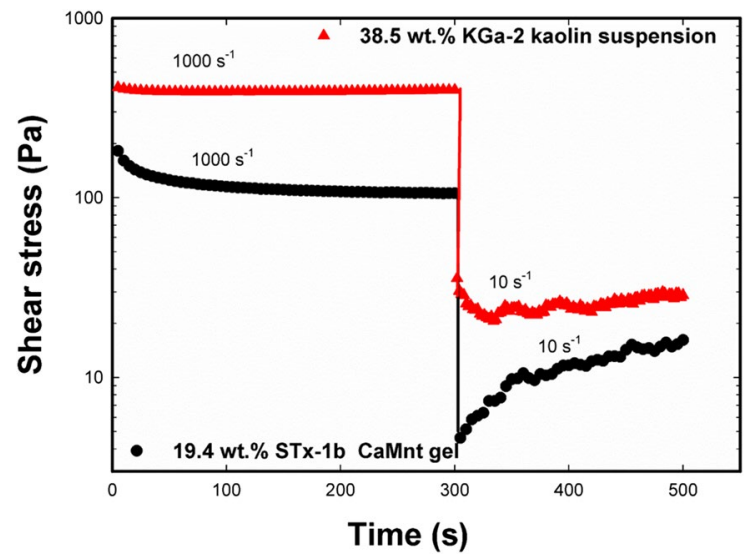

Fig. 8 A stepdown shear-rate method for determining whether a suspension or gel is time-dependent comprised of many platelets aligned in the shear flow direction. The abrupt decrease in shear rate caused the structure to reorganize to the new equilibrium state. Platelet-platelet repulsion drives this reorganization. The immediate and sustained shear stress increase after stepdown showed that the CaMnt gel is very time-dependent. In contrast, the 38.5 wt.\% kaolin suspension displayed no immediate increase in the shear stress after stepdown. Attractive force between the kaolin particles governed the structure formed.

However, both CaMnt and kaolin suspensions displayed a decreasing shear stress or viscosity with time at $1000 \mathrm{~s}^{-1}$. The decrease was pronounced for the CaMnt gel and small for the kaolin suspension. By definition, both of these suspensions are thixotropic (Guven, 1992), but the kaolin suspension was so weakly thixotropic that the structural recovery mode was not observed over the time span of the experiments. Upon stepdown to $10 \mathrm{~s}^{-1}$, the CaMnt gel displayed immediate rheopectic behavior, an increasing shear stress or viscosity with time. All thixotropic suspensions displayed rheopectic behavior upon stepdown in shear. The gel needed to develop a stronger structure at low shear rate so that it was able to display thixotropic behavior upon shearing at a higher rate. The kaolin suspension just needed a longer time for the stress to grow to a significant value at the low shear rate. The weak EDL repulsion is the cause.

\section{Conclusions}

At a concentration of $\sim 12$ wt.\% solids, an STx- $1 \mathrm{~b}$ CaMnt suspension at its natural $\mathrm{pH}$ of $\sim 8$ and conductivity did not display time-dependent behav-

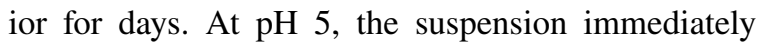
became time dependent. The face-face repulsion was enhanced as a result of an increase in the negative charge density of the face. The positively charged $\mathrm{Ca}^{2+}$ hydrolysis product adsorbed was eliminated from the platelet face at $\mathrm{pH}$ 5. It became fully hydrated and displaced to the solution. At higher solids loadings, time-dependent behavior was observed at $\mathrm{pH} 8$ despite the lower charge density of the face. The increased proximity of the interacting platelets allowed strong face-face EDL repulsion to operate and reorganize the structure. The 
particle morphology, low permanent layer charge, and the microstructure explained the time-independent behavior of KGa-2 kaolin suspension. The need for a strong EDL face-face repulsion for immediate thixotropic behavior of a 3-D gel network was further validated by the kaolin suspension results.

Acknowledgments Pengfei Liu thanks the Australian Research Training Program for providing the financial support for the PhD study. The authors acknowledge technical assistance from Lyn Kirilak and use of the facilities at Microscopy Australia at the Centre for Microscopy, Characterization \& Analysis, The University of Western Australia, a facility funded by the University, State, and Commonwealth Governments. The authors thank the reviewers, associate editor, and editor-in-chief for making this a better paper.

Funding Open Access funding enabled and organized by CAUL and its Member Institutions. Funding sources are as stated in the Acknowledgments.

\section{Declarations}

Conflict of Interest The authors declare that they have no conflict of interest.

Open Access This article is licensed under a Creative Commons Attribution 4.0 International License, which permits use, sharing, adaptation, distribution and reproduction in any medium or format, as long as you give appropriate credit to the original author(s) and the source, provide a link to the Creative Commons licence, and indicate if changes were made. The images or other third party material in this article are included in the article's Creative Commons licence, unless indicated otherwise in a credit line to the material. If material is not included in the article's Creative Commons licence and your intended use is not permitted by statutory regulation or exceeds the permitted use, you will need to obtain permission directly from the copyright holder. To view a copy of this licence, visit http://creativecommons.org/licenses/by/4.0/.

\section{References}

Abend, S., \& Lagaly, G. (2000). Sol-gel transitions of sodium montmorillonite slurries. Applied Clay Science, 16, 201-227.

Abu-Jdayil, B. (2011). Rheology of sodium and calcium bentonite-water dispersions: Effect of electrolytes and aging time. International Journal of Mineral Processing, 98, 208-213.

Au, P. I., \& Leong, Y. K. (2016). Surface chemistry and rheology of slurries of kaolinite and montmorillonite from different sources. Kona Powder and Particle Journal, 33, $17-32$.
Au, P. I., Clode, P., Smart, R. S. C., \& Leong, Y. K. (2015a). Surface chemistry-microstructure-rheology of high and low crystallinity KGa-1b and $\mathrm{KGa}-2$ kaolinite suspensions. Colloids and Surfaces A: Physicochemical and Engineering Aspects, 484, 354-364.

Au, P. I., Pillai, P., \& Leong, Y. K. (2015b). Ageing and collapse of bentonite gels - effects of $\mathrm{Mg}(\mathrm{II}), \mathrm{Ca}(\mathrm{II})$ and $\mathrm{Ba}(\mathrm{II})$ ions. Applied Clay Science, 114, 141-150.

Baes, C. F., \& Mesmer, R. E. (1986). The Hydrolysis of Cations. Kreiger.

Brandenburg, U., \& Lagaly, G. (1988). Rheological properties of sodium montmorillonite dispersions. Applied Clay Science, 3, 263-279.

Broughton, G., \& Squires, L. (1936). The Gelation of Bentonite Suspensions. Journal of Physical Chemistry, 40, 1041-1053.

Callaghan, I., \& Ottewill, R. (1974). Interparticle forces in montmorillonite gels. Faraday Discussions of the Chemical Society, 57, 110-118.

Choo, H. W., Choi, Y. M., Lee, W. J., \& Lee, C. H. (2020). Effect of $\mathrm{pH}$ Variations on the Yield Stress of Calcium Bentonite Slurry Treated with $\mathrm{pH}$-Responsive Polymer. Materials, 13, 2525.

Coussot, P., Nguyen, Q. D., Huynh, H., \& Bonn, D. (2002). Viscosity bifurcation in thixotropic, yielding fluids. Journal of Rheology, 46, 573-589.

Coussot, P., Tabuteau, H., Chateau, X., Tocquer, L., \& Ovarlez, G. (2006). Aging and solid or liquid behavior in pastes. Journal of Rheology, 50, 975-994.

Du, M., Liu, J., Clode, P. L., \& Leong, Y. K. (2018). Surface chemistry, rheology and microstructure of purified natural and synthetic hectorite suspensions. Physical Chemistry Chemical Physics, 20, 19221-19233.

Guven, N. (1992). Rheological aspects of aqueous smectite suspensions. In: N. Guven \& M. Pollastro (Eds), Claywater Interface and its Rheological Implications, CMS Workshop Lectures Vol. 4, The Clay Minerals Society, Boulder (pp 81-125).

Hauser, E., \& Reed, C. (1937). Studies in thixotropy. II. The thixotropic behavior structure of bentonite. Journal of Physical Chemistry, 41, 911-934.

Heller, H., \& Keren, H. (2001). Rheology of Na-rich montmorillonite suspension as affected by electrolyte concentration and shear rate. Clays and Clay Minerals, 49, 286-291.

Keren, R. (1988). Rheology of aqueous suspension of sodium/calcium montmorillonite. Soil Science Society of America Journal, 52, 924-928.

Lagaly, G. (2006). Colloid Clay Science. In F. Bergaya, B. K. G. Theng, \& G. Lagaly (Eds.), Handbook of Clay Science (Vol. 1, pp. 141-245). Elsevier Science.

Lee, C. E. J., Chandra, S., \& Leong, Y. K. (2012). Structural recovery behavior of kaolin, bentonite and K-montmorillonite slurries. Powder Technology, 223, 105-109.

Leong, Y. K., Du, M., Au, P. I., Clode, P., \& Liu, J. (2018). Microstructure of sodium montmorillonite gels with long aging time scale. Langmuir, 34, 9673-9682.

Leong, Y. K., Liu, P., Clode, P., \& Liu, J. (2021). Ageing behaviour spanning months of $\mathrm{NaMt}$, hectorite and Laponite gels: Surface forces and microstructurea comprehensive analysis. Colloids and Surfaces 
A: Physicochemical and Engineering Aspects, 630, 127543.

M'Ewen, M. B., \& Pratt, M. I. (1957). The gelation of montmorillonite. Part 1. - The formation of a structural framework in sols of Wyoming bentonite. Transactions of the Faraday Society, 53, 535-547.

Rudolph, W. W., \& Irmer, G. (2013). Hydration of the calcium(II) ion in an aqueous solution of common anions $\left(\mathrm{ClO}_{4}^{-}, \mathrm{Cl}^{-}, \mathrm{Br}^{-}\right.$, and $\left.\mathrm{NO}_{3}^{-}\right)$. Dalton Transactions, 42, 3919-3935.

Sueto, J., \& Nakaishi, K. (1992). Thixotropic behaviour of sodium and calcium montmorillonite at salt concentration in the vicinity of $0.3 \mathrm{~N}$. Clay Science, 8, 349-353.

Teich-McGoldrick, S. L., Greathouse, J. A., Jové-Colón, C. F., \& Cygan, R. T. (2015). Swelling properties of montmorillonite and beidellite clay minerals from molecular simulation: comparison of temperature, interlayer cation, and charge location effects. Journal of Physical Chemistry C, 119, 20880-20891.
Tombacz, E., \& Szekeres, M. (2004). Colloidal behavior of aqueous montmorillonite suspensions: the specific role of $\mathrm{pH}$ in the presence of indifferent electrolytes. Applied Clay Science, 27, 75-94.

Van Olphen, H. (1955). Forces between suspended bentonite particles. Clays and Clay Minerals, 4, 204-224.

Wyss, H. M., Tervoort, E., Meier, L. P., Müller, M., \& Gauckler, L. J. (2004). Relation between microstructure and mechanical behavior of concentrated silica gels. Journal of Colloid and Interface Science, 273, 455-462.

Zavitsas, A. A. (2005). Aqueous solutions of calcium Ions: hydration numbers and the effect of temperature. Journal of Physical Chemistry B, 109, 20636-20640.

Zhu, S., Avadiar, L., \& Leong, Y. K. (2016). Yield stress- and zeta potential-pH behavior of washed $\alpha-\mathrm{Al}_{2} \mathrm{O}_{3}$ suspensions with relatively high $\mathrm{Ca}$ (II) and $\mathrm{Mg}$ (II) concentrations: Hydrolysis product and bridging. International Journal of Mineral Processing, 148, 1-8. 